\title{
Digital Sources of Food Purchasing Data for the Surveillance of Dietary Patterns
}

\author{
Katia Charland*, Hiroshi Mamiya and David Buckeridge \\ McGill University, Montreal, QC, Canada
}

\section{Objective}

To demonstrate the utility of automatically captured store-level (i.e. point-of-sale) food purchasing data for the surveillance of dietary patterns before and after interventions. We assessed the effects of two interventions in Montreal, Canada that were intended to reduce the consumption of sugary drinks.

\section{Introduction}

In Canada, the economic impact of unhealthy eating is estimated at $\$ 6.3$ billion annually and in the US the estimated cost is $\$ 87$ billion. Despite the critical need to identify effective diet-related interventions through empirical evaluation, public health practitioners and researchers lack timely access to representative data sources collected at a fine spatial and temporal resolution. Food surveys, for example, are costly, infrequent, delayed, and subject to biases [1].

The Nielsen Corporation collects data on food purchasing directly from scanners in grocery and convenience stores around the world. These data hold great potential for public health practice. We were interested in using these data to analyze purchases of regular (sugary) soda and water, before and after two interventions aimed at reducing sugary drink consumption. The first intervention, 'Gobes-tu ça', was a counter-advertising campaign targeting the age group with the highest consumption of soda, 12-17 year olds [2]. The second intervention, 'Sois-futé, bois santé', targeted elementary school students. Both began in the Fall of 2011 and ramped up over time.

\section{Methods}

Using Nielsen data, we determined the monthly number of $(240 \mathrm{ml})$ servings of soda and water purchased at 180 sampled convenience and grocery stores in Montreal, Canada, from 2008 to 2013. The outcomes were the number of soda and water servings purchased per month and the proportion of servings (out of water and soda) that were soda servings. We first identified significant breakpoints in both the soda and water series [3]. Then, to assess the relationship between the timing of the intervention and the purchasing outcomes, we used log-linear and logistic time series regression. Heteroskedasticity and autocorrelation consistent estimators were used to account for temporal correlation [3]. The independent variable representing the timing of the intervention, had a value of zero before the intervention (before September 2011). Afterwards, to account for the intervention ramping up, the variable had a value equal to the number of months since the intervention was implemented. Covariates were the month, the average monthly maximum temperature, and the ratio of the average price of soda to water.

\section{Results}

RESULTS The number of soda servings sold and the proportion that were soda dropped in late 2011 (Figure). There was one significant breakpoint for the soda series in Dec 2011 (breakpoint month: 12/2011; 95\% confidence interval 3/2011, 12/2012) and none in the water series. After accounting for the average price ratio of soda to water, the month and the average maximum temperature, from the time of the intervention to the end of the study period there was an average $26 \%$ increase in water sales $(1.26,95 \%$ CI $1.09,1.46)$ and
$12 \%$ decrease in soda sales $(0.88 ; 95 \%$ CI $0.78,0.97)$, resulting in an average $30 \%$ decrease in the proportion of sales of soda (odds ratio $0.70 ; 95 \%$ CI $0.61,0.95)$

\section{Conclusions}

Using digital food purchasing data we identified a significant drop in the sales of soda relative to water following the public health interventions. Future work will involve examining geographical variations in the effect of the intervention, possibly due to socioeconomic status or demographic factors.

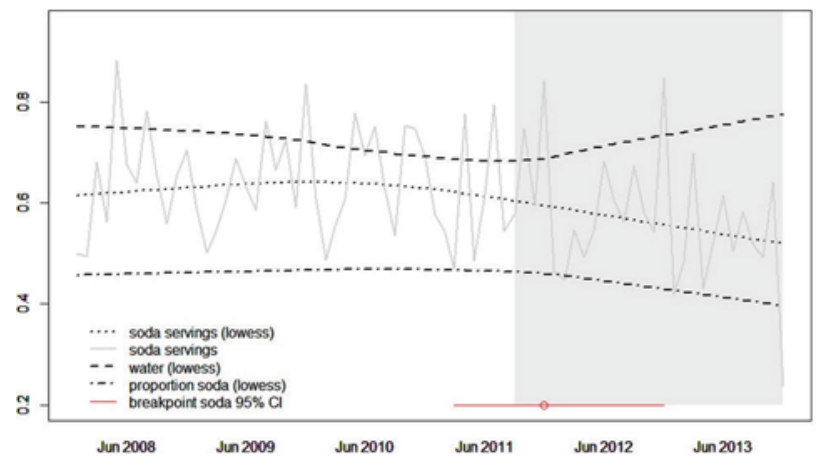

Figure: Monthly sales (/10 million) of soda, water, and the proportion of soda sales. The shaded region indicates the intervention period.

\section{Keywords}

Evaluation; Diet; Intervention

\section{References}

1. Diane McAmmond and Associates. Food and Nutrition Surveillance in Canada: An Environmental Scan. 2000 Mar.

2. Langlois K, Garriguet D. Sugar consumption among Canadians of all ages. Health Rep. 2011;22.

3. Zeileis A. Econometric Computing with $\mathrm{HC}$ and HAC Covariance Matrix Estimators. J Stat Softw. 2004 Nov; 11(10).

\section{*Katia Charland \\ E-mail: charlandk@yahoo.com}

